over the right side of the face without facial weakness. Right-sided cerebellar disturbance was indicated by ataxia and dysmetria in the routine tests performed with the right arm and leg.

The cerebrospinal fluid obtained by lumbar puncture showed a normal pressure with xanthochromia. Two days later, the neurological symptoms deteriorated gradually and the patient was confused or stuporous. Six days after admission, the right frontal ventricular tap with closed system drainage was performed. Seven days after admission, the pneumoventriculographic study showed a defect in the rhomboid fossa with displacement of the fourth ventricle to the left.

A suboccipital craniectomy was carried out. We found a bluish-colored elevation in the right and middle lower portion of the floor of the fourth ventricle, and a hematoma, semifluid collection of $18 \mathrm{cc}$ black blood, was evacuated. No tumor tissue was found, and the biopsies from the hematoma cavity were also negative.

Recovery was very satisfactory with slightly right-sided cerebellar disturbance and he was discharged two months after the operation. At the time of this report, improvement has been maintained for approximately one year and eleven months.

The pathogenesis of these primary hemorrhages of the pontien-medeullary part is, as shown by others, possibly related to a microaneurysm of a branch of the basilar artery.

\title{
54. The New Operative Procedure for the Garotid-Gavernous Fistula without any Interference to Blood Circulation of Internal Carotid Artery
}

\author{
Yuji Miyazaki, Sumiyoshi Tanabe, \\ Ishi Shimizu, Seisuke Akagawa \\ Department of Neurological Surgery, \\ Sapporo Medical College and Hospital
}

The main operative procedure for the carotid-cavernous fistula at present in general is trapping method developed by Hamby and Gardner. The trapping method has always possibility of cerebral circulation disturbance and visual failure postoperatively and has always unreliability in its effect.

The authors did experimental study to develope a new operative procedure which is able to close the fistula selectively without accompanying any interference of carotid circulation.

The priciple of the authors' new operative procedure is that the carotid-cavernous fistula is closed by plug with small piece of muscle embolus selectively. This small muscle embolus anchored in exact place by mean of thread attached to the embolus. 
The authors made a fistula between iliac artery and vein in dogs experimentally and the muscle embolus attached silk thread inserted into iliac artery. The position of muscle embolus was controlled by prolongation of length of thread in the artery like kit-flying. The artificial arteriovenous fistula was closed completely in these experimental studies.

Three cases of traumatic carotid-cavernous fistula were operated by this new operative procedure. The small muscle embolus with long thread were inserted into the internal carotid artery via external carotid artery. The distance of flow of muscle embolus in the internal carotid artery to the site of fistula was controlled by the length of thread from incised portion of external carotid artery to muscle embolus and flow of muscle embolus to the site of fistula was allowed by gradual loosening of holding thread under repeated check of position of silver clip placed the muscle embolus and repeated carotid angiography.

All subjective complaint disappeared immediately when muscle embolus reached in the fistula and the proptosis and congestion of eye disappeared within three days.

The follow up study in these cases were done and the longest follow up was 15 months without any sequela including cerebral thrombosis.

\title{
55. Carotid-Cavernous Fistula. Report of 10 Cases with the Usage of Newly Developed Plastic Emboli and Etiological Consideration of the Cases in Spontaneous Origin
}

\author{
Tomio Ohta, M. D., Shuro Nishimura, M. D., \\ Yoshinobu Mrki, M. D., Takashi Ozaki, M. D., \\ Junsuke Katsuyama, M. D., Kiyoharu Tanaka, M. D., \\ Haruhiko Kikuchi, M. D., Hiroshi Matsumura, M. D., \\ Akikazu ImaI, M. D., Masao RaI, M. D., \\ Koichro Hida, M. D., Mitsuro Toyama, M. D., and \\ Masayuki Fukuma, M. D. \\ Department of Neurosurgery, Osaka City University Med. \\ School, Osaka; \\ Department of Neurosurgery, Tenri Hospital, Tenri; \\ Department of Neurosurgery \\ Amagasaki Municipal Hospital, Amagasaki; \\ and Division of Neurosurgery, \\ First Surgical Department, Kyoto Prefectural Med. \\ School, Kyoto
}

Surgical treatments in 10 cases of the carotid-cavernous fistula have revealed remarkable difference in the surgical prognosis between the traumatic and the spon- 